\title{
Conciliación de la medicación al alta hospitalaria en farmacia comunitaria: a propósito de un caso
}

\author{
Ana Molinero ${ }^{1,2,3}$, Rebeca Escribano-Molinero ${ }^{3,4}$ \\ 1. Doctora en Farmacia. 2. Profesora Asociada del departamento de Ciencias Biomédicas de la Universidad de Alcalá (UAH). 3. Farmacéutica \\ comunitaria en Fuenlabrada (Madrid). 4. Graduada en Farmacia.
}

\section{PALABRAS CLAVE}

Conciliación de la medicación, farmacia comunitaria, error de medicación, servicios profesionales farmacéuticos

\section{ABREVIATURAS}

\section{D: discrepancia}

DJ: discrepancia justificada

DNJ: discrepancia no justificada

EC: error de conciliación

EM: error de medicación

FA: fibrilación auricular

FC: farmacia comunitaria

FV: fibrilación ventricular

HBPM: heparina de bajo peso molecular

INR: International Normalized Ratio

ITU: infección del tracto urinario

MAP: médico de atención primaria

MSI: miembro superior izquierdo

NICE: National Institute for Clinical Excellence

SEFH: Sociedad Española de Farmacia

Hospitalaria

SPF: Servicio Profesional Farmacéutico

\section{KEYWORDS}

Medication reconciliation, community pharmacy, medication error, pharmaceutical services

\section{RESUMEN}

Mujer de 86 años (itinerante entre dos comunidades autónomas) con fibrilación auricular (FA) persistente y fibrilación ventricular (FV) controlada con anticoagulación oral, distimia, desorden de ansiedad, temblor esencial e insuficiencia venosa crónica que acude a la farmacia comunitaria (FC) después de haber acudido a la consulta de su médico de atención primaria (MAP) y que ha sido diagnosticada al alta hospitalaria (en la otra comunidad autónoma) de ictus isquémico parietal derecho cardioembólico. No se ha realizado conciliación al alta y al dispensar las recetas del MAP se detecta que hay algunas duplicidades, faltan medicamentos prescritos en el informe de alta y sobran otros según lo que aparece en el informe al alta.

\section{Medication Reconciliation Upon Hospital Discharge Within Community Pharmacy: A Case Study}

\begin{abstract}
An 86-year-old female (itinerant between two autonomous regions) with persistent atrial fibrillation (AF) with controlled ventricular fibrillation (VF) with oral anticoagulation, dysthymia, anxiety disorder, essential tremor and chronic venous insufficiency arrives to the community pharmacy after visiting her primary care physician (PCP). She has been diagnosed at hospital discharge (in another region) with ischemic parietal cardioembolic ictus. No reconciliation was made to the discharge and when dispensing the prescriptions of the MAP we detected that there are some duplicities, there are prescriptions that are not in the discharge report and there are no prescriptions for many others according to what appears in the discharge report.
\end{abstract}

Este trabajo fue presentado en el VII Congreso Nacional de Farmacéuticos Comunitarios, Zaragoza 2016.

\section{Presentación del caso}

Se trata de una paciente de 86 años de edad, con domicilio itinerante, cada dos meses, entre sus dos hijos que viven en dos comunidades autónomas diferentes y que no mantienen comunicación entre ellos.

La paciente está diagnosticada desde hace años de asma bronquial persistente, fibrilación auricular (FA) per- sistente y fibrilación ventricular (FV) controlada con anticoagulación oral, distimia y trastorno de ansiedad generalizado, temblor esencial, osteoporosis, insuficiencia venosa crónica e infección del tracto urinario de repetición (ITU).

Acude a la farmacia comunitaria solicitando la dispensación de varias recetas médicas después de haber visitado al médico de atención primaria
(MAP) de nuestra localidad y con el informe del alta hospitalaria (en otra comunidad autónoma) en el que podemos leer como diagnóstico ictus isquémico parietal derecho cardioembólico.

Ingresa en el hospital porque la tarde del ingreso refiere clínica súbita, estando sentada, de mareo con alteración del lenguaje y pérdida de fuerza

Contribución a la autoría: las dos autoras contribuyeron por igual al diseño del estudio, el desarrollo de la investigación, el análisis de los resultados, la redacción del manuscrito y la revisión del contenido final.

Cite este artículo como: Molinero A, Escribano-Molinero R. Conciliación de la medicación al alta hospitalaria en farmacia comunitaria: a propósito de un caso. Farmacéuticos Comunitarios. 2017 Sep 30; 9(3):13-17. doi:10.5672/FC.2173-9218.(2017/ Vol9).003.03 
en el hemicuerpo izquierdo así como desviación de la comisura bucal.

Según leemos en el informe médico los familiares de la paciente refieren que al ingreso hospitalario toma la siguiente medicación:

Paracetamol y metamizol a demanda. Furosemida $40 \mathrm{mg}$ 1-0-0, digoxina $0,25 \mathrm{mg}^{1 / 2}-\mathrm{-}-0-0$, bisoprolol 2,5 mg $1 / 2-0-$ 0, Foster $\mathrm{Nxh}^{\circledR}$ 100/6 (beclometasona/ formoterol) 1-0-0, Ultibrobreezhaler ${ }^{\circledR}$ 85/43 (indacaterol/glicopirronio) 1-0-

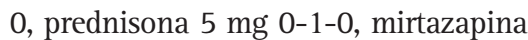
flas $15 \mathrm{mg}$ 1-0-0, Trankimazin $\mathrm{R}^{\circledR} 0,5$ mg (alprazolam retard) 0-0-1, Lyrica ${ }^{\circledR}$ $25 \mathrm{mg}$ (pregabalina) 1-1-1, Deprax ${ }^{\circledR}$ $100 \mathrm{mg}$ (trazodona) 0-0-0-1/2, lorazepam $1 \mathrm{mg}$ 0-0-0-1, Nexium ${ }^{\circledR} 20 \mathrm{mg}$ (esomeprazol) 1-0-1, simvastatina 40 mg 0-0-1, Sintrom ${ }^{\circledR} 4 \mathrm{mg}$ (acenocumarol) según calendario y Deltius ${ }^{\circledR} 25.000$ UI (colecalciferol) mensual.

Entre las pruebas de coagulación observamos INR (International Normalized Ratio) $=1,18$ y resto normal. Pruebas bioquímicas: glucosa, perfil renal, creatin-kinina y troponina dentro del rango de la normalidad.

Permanece ingresada durante cinco días con mejoría progresiva, persistiendo al alta paresia en miembro superior izquierdo (MSI) 2/5. Se trata de un ictus en relación a la FA por lo que se retrasa de nuevo el inicio de la anticoagulación al menos siete días para evitar hemorragias secundarias.

El diagnóstico al alta es: ictus isquémico parietal derecho de origen posiblemente cardioembólico, infección urinaria por Escherichia coli (pendiente de confirmación por laboratorio) y leve ferropenia.

El tratamiento recomendado, con firma del médico responsable, en el informe médico al alta es:

- Fluidoterapia y nutrición. Dieta sosa sin grasas y baja en azúcares refinados.

- Movilización precoz. Rehabilitación del MSI (movilización del miembro con frecuencia).

- Clexane ${ }^{\circledR} 60$ mg (enoxaparina) jeringa precargada (0-1-0) durante 5 dias, continuar con apixaban $2,5 \mathrm{mg}$ (1-0-0), atorvastatina $40 \mathrm{mg}$ (0-0-1), Fero-gradumet ${ }^{\circledR} \quad$ (hierro sulfato): 1 comprimido al día durante 7 días al mes. Suspender simvastatina y Sintrom ${ }^{\circledR}$ (acenocumarol). Resto de medicación igual.

La hija de la paciente nos aporta las siguientes recetas en formato papel informatizadas del MAP local realizadas unos minutos antes:

Furosemida $40 \mathrm{mg}$, Digoxina Kern ${ }^{\circledR} 0,25 \mathrm{mg}$, Sintrom ${ }^{\circledR} 4 \mathrm{mg}$, simvastatina $40 \mathrm{mg}$, atorvastatina $40 \mathrm{mg}$, mirtazapina flas $30 \mathrm{mg}$, Trankimazin $\mathrm{R}^{\circledR} 0,5 \mathrm{mg}$, Lyrica ${ }^{\circledR} 25 \mathrm{mg}$ trazodona $100 \mathrm{mg}$, lorazepam $1 \mathrm{mg}$ y amlodipino $5 \mathrm{mg}$.

La hija nos dice que "le parece que no le han recetado todo lo que necesita su madre" y nos aporta también una hoja de medicación, que no ha enseñado al MAP local, de un tratamiento prescrito por un psiquiatra privado de la comunidad autónoma en la que la madre ha vivido los últimos dos meses, en la que leemos: paroxetina 20 mg 1-0-0, Lyrica ${ }^{\circledR} 25$ mg 1-1-1, Deprax ${ }^{\circledR} 100$ mg 0-0-0-1/2, mirtazapina flas $15 \mathrm{mg} 0-0-1$, Trankimazin $\operatorname{Retard}^{\circledR}$ $0,5 \mathrm{mg}$ 0-0-1. Si aparece angustia o ataque de pánico un comprimido disuelto en la boca de Zyprexa ${ }^{\circledR}$ Velotab (olanzapina) $5 \mathrm{mg}$ una vez al día.

\section{Objetivos}

Realizar una continuidad asistencial en la farmacia comunitaria mediante la evaluación y conciliación de la medicación de un paciente tras el alta hospitalaria.

\section{Evaluación}

Procedemos a realizar un Servicio Profesional Farmacéutico (SPF) de Conciliación, definido por Foro de Atención Farmacéutica en Farmacia Comunitaria [1] y la Sociedad Española de Farmacia Hospitalaria (SEFH) como: la conciliación de la medicación en farmacia comunitaria es el servicio profesional en el que el farmacéutico realiza una comparación sistemática y protocolizada de la lista de medicamentos utilizados por el paciente, antes y después de una transición entre niveles asistenciales, con el objetivo de asegurar la necesidad, efectividad y seguridad del tratamiento farmacológico actual realizando las oportunas intervenciones, en coordinación con los profesionales sanitarios implicados. La actuación principal de este servicio consiste en la identificación, por parte del farmacéutico comunitario, de posibles discrepancias para su posterior análisis, evaluación y resolución, en su caso.

El objetivo final es reducir los efectos adversos de los medicamentos y evi- tar los errores de medicación (EM) [2].

Aplicamos el procedimiento diseñado en nuestra farmacia para el servicio de conciliación al alta hospitalaria sin conciliación en el hospital, en el que hemos adaptado las definiciones de la SEFH [3]:

- Discrepancia (D): cualquier diferencia entre la medicación habitual que el paciente tomaba previamente y la medicación prescrita después de una transición asistencial

- Discrepancia justificada (DJ): discrepancia que se explica con la información contenida en el informe de alta o con la información obtenida en la entrevista con el paciente, o discrepancia que requiere aclaración y el prescriptor no modifica en el tratamiento una vez informado de ésta.

- Discrepancia que requiere aclaración: discrepancia que no se explica, a priori, por la situación clínica del paciente y requiere una consulta al médico responsable del paciente.

- Discrepancia no intencionada o error de conciliación (EC): discrepancia que requiere aclaración y tras ser comunicada al médico es corregida en la prescripción.

- Discrepancia pendiente de resolución: discrepancia que requiere aclaración pero no se ha podido contactar con el prescriptor o no se ha obtenido respuesta.

Utilizamos un algoritmo de conciliación de la medicación adaptado del programa "Concilia Medicamentos" [4] y basado en la guía NICE [5] para la optimización de la medicación (figura 1).

Después del análisis obtenemos 15 discrepancias (tabla 1) al comparar la medicación activa de la paciente después del paso por el MAP local con la medicación al ingreso hospitalario, al alta y con el informe del psiquiatra de la medicina privada.

\section{Intervención}

La anticoagulación profiláctica tras un accidente cerebrovascular está justificada en pacientes con alto riesgo de trombosis venosa profunda y se considerará de elección la utilización de heparinas de bajo peso molecular (HBPM) (entre ellas enoxaparina) [6].

La sustitución del anticoagulante acenocumarol por apixaban está justificada para la prevención del ictus y la embolia sistémica en pacientes con FA y al menos un factor de riesgo 


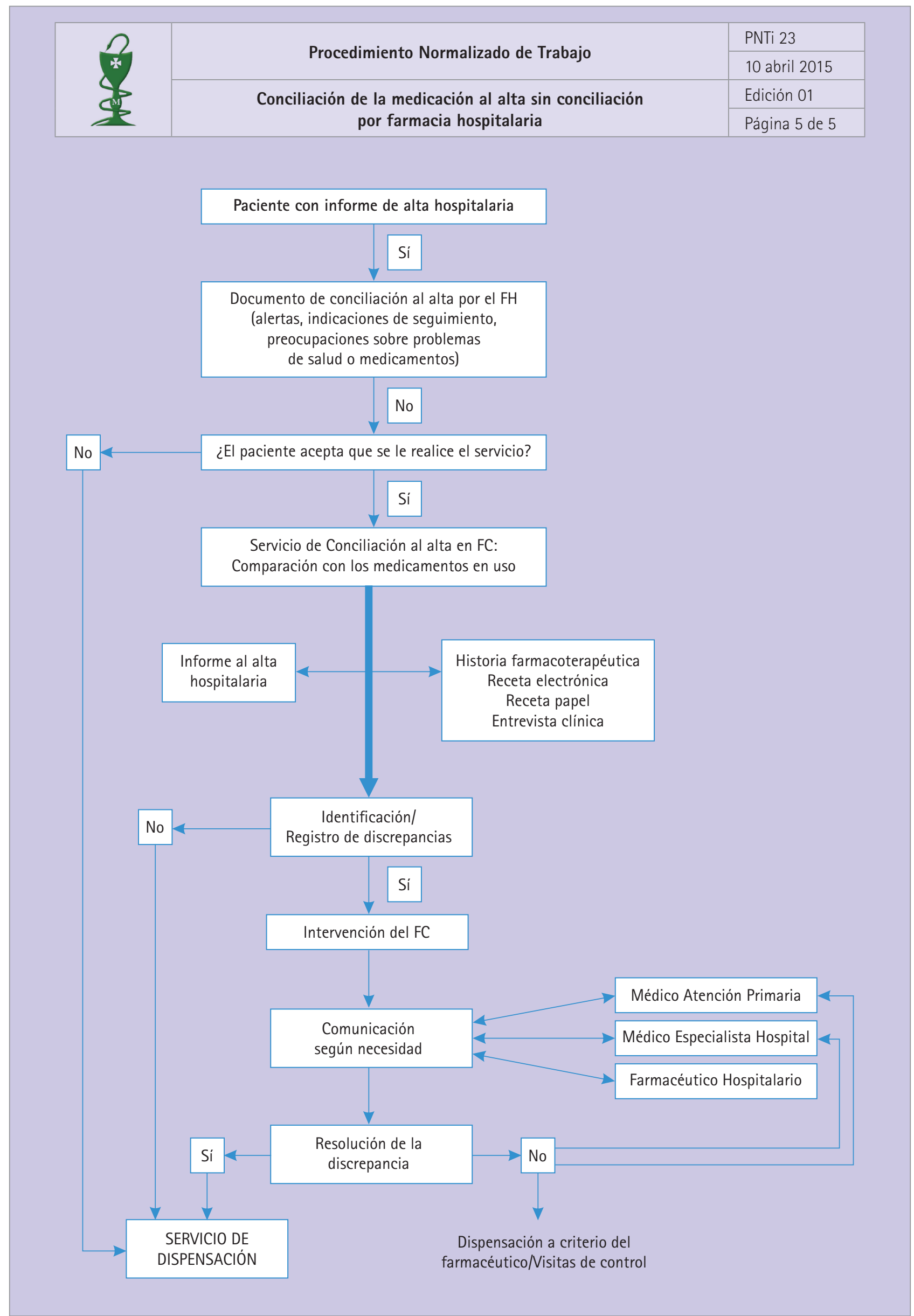

Figura 1 Algoritmo de conciliación de la medicación al alta adaptado de "Concilia Medicamentos" [4] 
Tabla 1 Discrepancias encontradas

\begin{tabular}{|c|c|c|c|}
\hline \multicolumn{4}{|c|}{ Paciente XXXXXXXXXXXX Fecha 04/12/2015 } \\
\hline \multirow[t]{2}{*}{$\begin{array}{l}\text { Prescripción al alta } \\
\text { hospitalaria }\end{array}$} & $\begin{array}{c}\text { Prescripción al ingreso } \\
\text { hospitalario } \\
\text { (referido por la paciente) }\end{array}$ & $\begin{array}{c}\text { Prescripción en receta papel } \\
\text { por MAP }\end{array}$ & $\begin{array}{l}\text { Prescripción de médico } \\
\text { privado (psiquiatra) }\end{array}$ \\
\hline & $\begin{array}{l}\text { Acenocumarol } 4 \text { mg } \\
\text { (según pauta) }\end{array}$ & $\begin{array}{l}\text { Acenocumarol } 4 \mathrm{mg} \\
\text { (según pauta) }\end{array}$ & \\
\hline \multicolumn{4}{|l|}{$\begin{array}{l}\text { Enoxaparina } 60 \text { mg (6.000 UI) } \\
10 \text { jeringas }(0-1-0)\end{array}$} \\
\hline \multicolumn{4}{|l|}{$\begin{array}{l}\text { Apixaban } 2,5 \mathrm{mg} \\
(1-0-0)\end{array}$} \\
\hline \multirow[t]{2}{*}{$\begin{array}{l}\text { Atorvastatina } 40 \mathrm{mg} \\
(0-0-1)\end{array}$} & & $\begin{array}{l}\text { Atorvastatina } 40 \mathrm{mg} \\
(0-0-1)\end{array}$ & \\
\hline & $\begin{array}{l}\text { Simvastatina } 40 \mathrm{mg} \\
(0-0-1)\end{array}$ & $\begin{array}{l}\text { Simvastatina } 40 \mathrm{mg} \\
(0-0-1)\end{array}$ & \\
\hline \multicolumn{4}{|l|}{$\begin{array}{l}\text { Hierro Sulfato } 105 \mathrm{mg} \\
(1-0-0)\end{array}$} \\
\hline $\begin{array}{l}\text { Furosemida } 40 \mathrm{mg} \\
(1-0-0)\end{array}$ & $\begin{array}{l}\text { Furosemida } 40 \mathrm{mg} \\
(1-0-0)\end{array}$ & $\begin{array}{l}\text { Furosemida } 40 \mathrm{mg} \\
(1-0-0)\end{array}$ & \\
\hline $\begin{array}{l}\text { Digoxina } 0,25 \mathrm{mg} \\
(1 / 2-0-0)\end{array}$ & $\begin{array}{l}\text { Digoxina } 0,25 \mathrm{mg} \\
(1 / 2-0-0)\end{array}$ & $\begin{array}{l}\text { Digoxina } 0,25 \mathrm{mg} \\
(1 / 2-0-0)\end{array}$ & \\
\hline $\begin{array}{l}\text { Bisoprolol } 2,5 \mathrm{mg} \\
(1 / 2-0-0)\end{array}$ & $\begin{array}{l}\text { Bisoprolol } 2,5 \mathrm{mg} \\
(1 / 2-0-0)\end{array}$ & & $\begin{array}{l}\text { Bisoprolol } 2,5 \mathrm{mg} \\
(1 / 2-0-0)\end{array}$ \\
\hline $\begin{array}{l}\text { Beclometasona/Formoterol } \\
100 / 6 \text { Nxh (1-0-0) }\end{array}$ & $\begin{array}{l}\text { Beclometasona/Formoterol } \\
100 / 6 \text { Nxh (1-0-0) }\end{array}$ & & \\
\hline $\begin{array}{l}\text { Indacaterol/Glicopirronio } \\
85 / 42(1-0-0)\end{array}$ & $\begin{array}{l}\text { Indacaterol/Glicopirronio } \\
85 / 42(1-0-0)\end{array}$ & & \\
\hline $\begin{array}{l}\text { Prednisona } 5 \mathrm{mg} \\
(0-1-0)\end{array}$ & $\begin{array}{l}\text { Prednisona } 5 \mathrm{mg} \\
(0-1-0)\end{array}$ & & \\
\hline $\begin{array}{l}\text { Mirtazapina Flas } 15 \mathrm{mg} \\
(1-0-0)\end{array}$ & $\begin{array}{l}\text { Mirtazapina Flas } 15 \mathrm{mg} \\
(1-0-0)\end{array}$ & $\begin{array}{l}\text { Mirtazapina Flas } 30 \mathrm{mg} \\
(1-0-0)\end{array}$ & $\begin{array}{l}\text { Mirtazapina Flas } 15 \mathrm{mg} \\
(0-0-1)\end{array}$ \\
\hline $\begin{array}{l}\text { Trazodona } 100 \mathrm{mg} \\
(0-0-0-1 / 2)\end{array}$ & $\begin{array}{l}\text { Trazodona } 100 \mathrm{mg} \\
(0-0-0-1 / 2)\end{array}$ & $\begin{array}{l}\text { Trazodona } 100 \mathrm{mg} \\
(0-0-0-1 / 2)\end{array}$ & $\begin{array}{l}\text { Trazodona } 100 \mathrm{mg} \\
(0-0-0-1 / 2)\end{array}$ \\
\hline $\begin{array}{l}\text { Esomeprazol } 20 \mathrm{mg} \\
(1-0-1)\end{array}$ & $\begin{array}{l}\text { Esomeprazol } 20 \mathrm{mg} \\
(1-0-1)\end{array}$ & & \\
\hline $\begin{array}{l}\text { Colecalciferol 25.000UI } \\
\text { (1 al mes) }\end{array}$ & $\begin{array}{l}\text { Colecalciferol 25000UI } \\
\text { (1 al mes) }\end{array}$ & & \\
\hline $\begin{array}{l}\text { Alprazolam Retard 0,5 mg } \\
(0-0-1)\end{array}$ & $\begin{array}{l}\text { Alprazolam Retard 0,5 mg } \\
(0-0-1)\end{array}$ & $\begin{array}{l}\text { Alprazolam Retard } 0,5 \mathrm{mg} \\
(0-0-1)\end{array}$ & $\begin{array}{l}\text { Alprazolam Retard 0,5 mg } \\
(0-0-1)\end{array}$ \\
\hline $\begin{array}{l}\text { Pregabalina } 25 \mathrm{mg} \\
(1-1-1)\end{array}$ & $\begin{array}{l}\text { Pregabalina } 25 \mathrm{mg} \\
(1-1-1)\end{array}$ & $\begin{array}{l}\text { Pregabalina } 25 \mathrm{mg} \\
(1-1-1)\end{array}$ & $\begin{array}{l}\text { Pregabalina } 25 \mathrm{mg} \\
(1-1-1)\end{array}$ \\
\hline \multirow[t]{4}{*}{$\begin{array}{l}\text { Lorazepam } 1 \mathrm{mg} \\
(0-0-0-1)\end{array}$} & $\begin{array}{l}\text { Lorazepam } 1 \mathrm{mg} \\
(0-0-0-1)\end{array}$ & $\begin{array}{l}\text { Lorazepam } 1 \mathrm{mg} \\
(0-0-0-1)\end{array}$ & \\
\hline & & $\begin{array}{l}\text { Amlodipino } 5 \mathrm{mg} \\
(1-0-0)\end{array}$ & \\
\hline & & & $\begin{array}{l}\text { Paroxetina } 20 \mathrm{mg} \\
(1-0-0)\end{array}$ \\
\hline & & & $\begin{array}{l}\text { Olanzapina velotabs } 5 \mathrm{mg} \\
(1-0-0)\end{array}$ \\
\hline
\end{tabular}

Discrepancias no intencionadas o errores de conciliación.

Discrepancias justificadas.

Discrepancias justificadas en base a la Historia Clínica de la paciente.

Discrepancias pendientes de resolución. 
adicional de complicaciones tromboembólicas principalmente por el estudio pivotal ARISTOTLE [7].

Además confirmamos mediante llamada telefónica al servicio de neurología del hospital donde ha estado ingresada la paciente que deben realizarse estos cambios, así como sustituir la simvastatina por la atorvastatina (discrepancias justificadas explicadas con las guías de práctica clínica e informe al alta hospitalaria).

También nos confirman que debe iniciar tratamiento con hierro, pero que éste puede instaurarse en unos días (discrepancias justificadas por aclaración con el servicio de neurología del hospital).

Por último, nos informan que la medicación referida al ingreso no está confirmada con ninguna prescripción médica y que ha sido anotada según lo que han comunicado los familiares de la paciente.

Comunicamos telefónicamente con el MAP de nuestra localidad y nos confirma que ha duplicado las estatinas y no debería haber prescrito acenocumarol $4 \mathrm{mg}$ (discrepancias no intencionadas o error de conciliación).

Bisoprolol 2,5 mg no aparece en la historia clínica del MAP local, por lo que intentamos hablar con el MAP del pueblo donde ha pasado la paciente los últimos dos meses sin obtener resultado positivo. También mantenemos conversación telefónica con el psiquiatra privado y nos comenta que en su historia clínica aparece que está en tratamiento con bisoprolol 2,5 mg (discrepancia pendiente de resolución).

Respecto a beclometasona/formoterol Nxh 100/6, indacaterol/glicopirronio 85/42 y prednisona $5 \mathrm{mg}$ nos indica la hija de la paciente que lo había tenido prescrito la primavera anterior por una crisis asmática que tuvo, pero que ahora no necesitaba tratamiento (discrepancias justificadas por aclaración con la hija de la paciente). Confirmado también con el MAP.

El psiquiatra privado nos confirma que la paciente está en tratamiento para la depresión con mirtazapina flas $15 \mathrm{mg}$ y paroxetina $20 \mathrm{mg}$ y si tiene angustia o ataques de pánico olanzapina velotabs $5 \mathrm{mg}$. El MAP local nos informa que la paciente solo está en tratamiento con mirtazapina flas $30 \mathrm{mg}$, pero que revisará de nuevo la medicación para determinar si necesita paroxetina $20 \mathrm{mg}$ o no (discrepancias que requieren aclaración).
El MAP local ha olvidado prescribir esomeprazol $20 \mathrm{mg}$, pero es necesario para la paciente (discrepancia no intencionada o error de conciliación).

La hija de la paciente no ve necesidad de la dispensación de colecalciferol 25.000 UI porque puede esperar (discrepancia justificada por aclaración con la hija de la paciente).

Respecto a amlodipino $5 \mathrm{mg}$ el MAP local nos confirma que la paciente lleva en tratamiento desde hace años con ese medicamento y que es necesario para el diagnóstico de hipertensión que tiene en su historia clínica (discrepancia justificada, aclarada por el MAP).

\section{Resolución de las discrepancias}

Se acuerda finalmente con su MAP no dispensar: acenocumarol $4 \mathrm{mg}$, simvastatina $40 \mathrm{mg}$, bisoprolol 2,5 mg, paroxetina $20 \mathrm{mg}$ y olanzapina velotabs $5 \mathrm{mg}$. Estos tres últimos medicamentos deben ser reevaluados por el MAP que cita a la paciente en su consulta para la semana siguiente. Y dispensar enoxaparina $60 \mathrm{mg}$, apixaban 2,5 mg y esomeprazol $20 \mathrm{mg}$. La hija de la paciente acudirá a su consulta para recoger las recetas médicas pertinentes.

\section{Resultado}

La conciliación de la medicación permitió que la paciente accediese en ese momento a la medicación que necesitaba y evitó la aparición de resultados negativos de la medicación (RNM) de seguridad (acenocumarol, simvastatina/atorvastatina) y de necesidad (enoxaparina, apixaban, esomeprazol).

El tiempo empleado por el farmacéutico para realizar la conciliación fue de 60 minutos.

A la semana siguiente el MAP reevaluó la medicación añadiendo Ultibro breezhaler ${ }^{\circledR}$ 85/43, bisoprolol 2,5 mg (1-0-0) y paroxetina $20 \mathrm{mg}$ (1-0-0) y cambió la dosis de mirtazapina flas a $15 \mathrm{mg}(0-0-0-1)$.

\section{Conclusiones}

El farmacéutico comunitario tiene acceso a toda la medicación que toma el paciente y mediante el uso de protocolos de conciliación puede identificar, analizar, evaluar y resolver reales o potenciales errores de medicación.

La comunicación entre diferentes profesionales, siempre que se produ- ce una transición asistencial, ayuda a minimizar errores de medicación en los pacientes y debe ser práctica habitual para evitar riesgos.

\section{Consideraciones éticas}

Las autoras han sido autorizadas a la difusión del caso por la paciente, manteniendo la anonimidad de la misma y la confidencialidad de los datos, siguiendo la Ley Orgánica de Protección de Datos (LOPD).

\section{Referencias bibliográficas}

1. Foro de Atención Farmacéutica en Farmacia Comunitaria, panel de expertos. Servicio de conciliación de la medicación en la farmacia comunitaria. [Internet]. Madrid: CGCOF; 2016. [Acceso 05-06-2017]. Disponible en: http://www.portalfarma.com/inicio/ serviciosprofesionales/forofarmaciacomunitaria/comisionconciliacion/Documents/FOLLETO-CONCILIACION

2. ISMP Medication Safety Alert! [Internet]. 2006 13th July. [Acceso 0506-2017]. Disponible en: http://www. ismp.org/newsletters/acutecare/articles/20060713.asp

3. Sociedad Española de Farmacia Hospitalaria. Documento de consenso en terminología y clasificación de los programas de Conciliación de la Medicación. Barcelona: Mayo; 2009.

4. Consejo General de Colegios Oficiales de Farmacéuticos. Concilia Medicamentos: Conciliación de la Medicación al alta hospitalaria en Farmacia Comunitaria. [Internet]. Madrid: CGCOF; 2017. [Acceso 05-06-2017]. Disponible en: http://www.portalfarma.com/ Profesionales/InvestigacionFarmacia/ Concilia/Documents/LIBRETO-CONCILIA-DIGITAL.PDF

5. National Institute for Clinical Excellence NICE. Medicines optimization: the safe and effective use of medicines to enable the best possible outcomes (NG5) [Internet]. Manchester: National Institute for Health and Care Excellence; 2015. [Acceso 12-1-2015]. Disponible en: https:// www.nice.org.uk/guidance/ng5

6. Lindsay MP, Gubitz G, Bayley M, Hill MD, Davies-Schinkel C, Singh S, et al. Canadian Best Practice Recommendations for Stroke Care (Update 2010). On behalf of the Canadian Stroke Strategy Best Practices and Standards Writing Group. Ottawa, Ontario Canada: Canadian Stroke Network; 2010.

7. Granger CB, Alexander JH, McMurray JJ, Lopes RD, Hylek EM, Hanna $M$ et al; ARISTOTLE Committees and Investigators. Apixaban versus warfarin in patients with atrial fibrillation. $\mathrm{N}$ Engl J Med. 2011; 365:981-992. doi:10.1056/NEJMoa1107039 